CATALAN REVIEW

Catalan Review

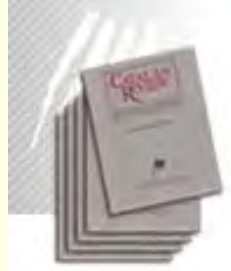

You are accessing the Digital Archive of the Catalan Review Journal.

By accessing and/or using this Digital Archive, you accept and agree to abide by the Terms and Conditions of Use available at http://www.nacs-

catalanstudies.org/catalan_review.html

Catalan Review is the premier international scholarly journal devoted to all aspects of Catalan culture. By Catalan culture is understood all manifestations of intellectual and artistic life produced in the Catalan language or in the geographical areas where Catalan is spoken. Catalan Review has been in publication since 1986 .
NORTH

AMERICAN

CATALAN

SOCIETY
Esteu accedint a l'Arxiu Digital del Catalan Review

A l' accedir i / o utilitzar aquest Arxiu Digital, vostè accepta i es compromet a complir els termes i condicions d'ús disponibles a http://www.nacs-

catalanstudies.org/catalan_review.html

Catalan Review és la primera revista internacional dedicada a tots els aspectes de la cultura catalana. Per la cultura catalana s'entén totes les manifestacions de la vida intel lectual i artística produïda en llengua catalana o en les zones geogràfiques on es parla català. Catalan Review es publica des de 1986.

\title{
Works by J. V. Foix and Selected Bibliography
}

Catalan Review, Vol. I, number 1, (1986), p. 327 -337 


\section{WORKS BY J. V. FOIX}

POETRY

Sol, $i$ de dol, Barcelona, Edicions L'Amic de les Arts, 1936.

Les irreals omegues, Barcelona, Edicions L'Amic de les Arts, 1949.

On he deixat les claus..., Barcelona, Edicions L'Amic de les Arts, 1953.

J. Ferraté - J. V. Foix, Cobles en bonor de J. V. Foix. Plant d'En Joseph Vicenç de Barcelona, en lo qual respòs a en Joan Ferreter, de Sent Jacme de Cuba, les cobles que li tramès; parla-y també d'En Joseph Roca e Ponç, de la metexa Universitat, from Inquietud, Vic, 1957.

Onze Nadals i un Cap d'Any, Barcelona, Edicions L'Amic de les Arts, 1960.

Desa aquests llibres al calaix de baix, Barcelona, Edicions L'Amic de les Arts, 1972.

\section{POETICAL PROSE}

Gertrudis, Barcelona, Edicions L'Amic de les Arts, 1927. KRTU, Barcelona, Edicions L'Amic de les Arts, 1932.

Còpia d'una lletra tramesa a Na Madrona Puignau, de Palau Ça Verdera, Barcelona, Dau al Set, 1951.

Del "Diari 1918", Barcelona, J. Horta, Editor, 1956 (preface by J. Romeu).

L'Estrella d'En Perris, Barcelona, Editorial Fontanella, 1963. 
Darrer comunicat, Barcelona, Edicions 62, 1970.

Allò que no diu "La. Vanguardia", Barcelona, Edicions Proa, 1970.

La pell de la pell, Barcelona, La Polígrafa, 1970 (illustrations by $\mathrm{J}$. Ponç).

Tocant a mà..., Barcelona, Edicions 62, 1972.

97 notes sobre ficcions poncianes, Barcelona, La Polígrafa, 1974 (illustrations by J. Ponç).

Quatre colors aparien el món, Barcelona, G. Gili, 1975 (illustrations by J. Miró).

97 notes sobre ficcions poncianes and La pell de la pell, Barcelona, La Magrana, 1977.

Diari 1918, Barcelona, Edicions 62, 1981.

Discurs de Josep V. Foix from Homenatge a Josep V. Foix amb motiu d'ésser-li conferit el grau de Doctor Honoris Causa, Barcelona, Universitat de Barcelona, 1984.

L'estació, Barcelonă, Taché editor, 1984 (illustrations by A. Tàpies).

Cròniques de l'ultrason and L'estació, Barcelona, Edicions L'Amic de les Arts-Quaderns Crema, 1985.

ARTICLES AND ESSAYS

Revalució catalanista (with the collaboration of J. Carbonell), Barcelona, Edicions Monitor, 1934.

$\mathrm{J}$. Verdaguer, Prosa antològica (selection and preface by $\mathrm{J}$. V. Foix), Barcelona, Ed. Selecta, 1952.

Catalans de 1918 (preface by J. Colomines), Barcelona, Edicions 62, 1965.

Els lloms transparents (preface by G. Ferrater), Barcelona, Edicions 62, 1969. 
Mots $i$ maons o a cascú el seu, Barcelona, Edicions L'Amic de les Arts, 1971.

\section{COLLECTED WORKS}

Obres poètiques, Barcelona, Editorial Nauta, 1964.

Obres completes, Barcelona, Edicions 62: Vol. I: Poesia (1974) (Preface by P. Gimferrer); Vol. II: Prosa (1979); Vol. III: Articles $i$ assaigs politics (1985) (Edited by M. Carbonell).

Obra poètica (Edited by Jaume VallcorbaPlana) Barcelona, Edicions dels Quaderns Crema: I: Gertrudis (1983); II: KRTU (1983); III: Sol, i de dol (1985); IV: Les irreals omegues (1986); VI: Onze nadals i un cap d'any (1984).

\section{ANTHOLOGIES}

Antología de J. V. Foix (Edited by E. Badosa), Barcelona, Plaza y Janés, S. A., 1969.

Antologia poètica, Barcelona, Edicions Proa, 1973.

J. V. Foix en els seus millors escrits (Edited by M. Arimany), Barcelona, Arimany, 1973.

Antologia poètica (Edited by P. Gimferrer), Barcelona, Edicions 62, 1980. 


\section{SELECTED BIBLIOGRAPHY}

In this selection we have not included articles that appeared in newspapers: we have only taken into consideration books an articles published in journals, taking the bibliography about Foix included in Faig (June 1984) and the one found in Patricia Boehne's J. V. Foix as basic points of reference. We have included also general studies about Catalan literature and Avantguardism by way of background to the more specialized writings on Foix, which will follow.

\section{A. General Studies about Catalan literature}

A. Carbonell, A. M. Espadaler, J. Llovet and A. TayadeIla, Literatura catalana. Dels inicis als nostres dies, Barcelona, Edhasa, 1979.

J. M. Castellet and J. Molas, Poesia catalana del segle XX, Barcelona, Edicions 62, 1963.

$\mathrm{J}$. Folguera, Les noves valors de la poesia actual, Barcelona, La Revista, 1919.

J. Fuster, La poesia catalana (2 volumes), Palma de Mallorca, Moll, 1956.

J. Fuster, Literatura Catalana Contemporània, Barcelona, Curial, 1972.

M. de Riquer and A. Comas, Història de la literatura catalana (6 volumes), Barcelona, Ariel, 1964-1985.

J. Roca-Pons, Introduction to Catalan Literature, Bloomington, Indiana U. Publications, 1977. 
J. Ruiz Calonja, Història de la literatura catalana, Barcelona, Teide, 1954.

A. Terry, Catalan Literature, London, Ernest Benn Ltd. and New York, Barnes and Noble Books, 1972.

J. Triadú, Antbology of Catalan Lyric Poetry, Berkeley, U. of California Press, 1953.

J. Triadú, Nova antologia de la poesia catalana (1900-1964), Barcelona, Selecta, 1965.

P. Verdaguer, Histoire de la littérature catalane, Barcelone, Barcino, 1981.

\section{B. STUDIES ON AVANTGUARDISM}

M. Arimany, L'avantguardisme en la poesia catalana actual, Barcelona, Arimany, 1972.

J. Arús, Les posicions extremes en l'art i en la literatura, Barcelona, Ariel, 1925.

A. Balakian, Literary Origins of Surrealism, New York, New York U. Press, 1947.

G. Díaz-Plaja, "Els moviments dits d'avantguarda a Catalunya (Notes per a un estudi)", L'avantguardisme a Catalunya $i$ altres notes de crítica, Barcelona, La Revista, 1932, 7-35.

Y. Duplessis, Le surréalisme, Paris, Presses Universitaires de France, 1967 ("Que sais-je", 432).

M. Durán, El superrealismo en la poesía española contemporánea, México, 1950.

M. Durán, "Love at First Sight: Spanish Surrealism Reconsidered", Modern Language Notes, LXXXIV, 1969.

P. Éluard, Qu'est-ce que le surréalisme?, Bruxelles, R. Henriques, 1934.

P. Éluard, Notes sur la poésie, Paris, G. L. M., 1936. 
J. M. Junoy, Obra poètica (Edited by J. VallcorbaPlana), Barcelona, Edicions dels Quaderns Crema, 1984.

C. B. Morris, Surrealism and Spain, Cambridge, Cambridge U. Press, 1972.

\section{WORKS ABOUT FOIX AND HIS WRITINGS}

J. Argenté-Giralt, La poesia de J. V. Foix. Dissertation read in the U. of Barcelona, 1958.

C. Armendares, J. V. Foix: La Seva Obra. Dissertation read in the U. of Paris (Sorbonne), 1972.

E. Badosa, "De la poesía religiosa de J. V. Foix", Destino, 1.037 (1957), 38.

E. Badosa, preface to his Antología lírica, Madrid, Rialp, 1963.

E. Badosa, preface to his Antología de J. V. Foix, Barcelona, Plaza y Janés, S. A., 1969.

P. Boehne, "J. V. Foix: The Poetic Search for Personal Reality", in J. Gulsoy and J. M. Solà-Solé, Catalan Studies. Volume in Memory of Josephine de Boer, Barcelona, Hispam, 1977.

P. Boehne, J. V. Foix, Boston, Twayne Publishers, 1980.

P. Boehne, "J. V. Foix: A Catalan Transformation of Reality", Discurso Literario (Vol. 2, No. 2), Oklahoma State U., Stillwater, Oklahoma (USA).

L. Bonet, "El realismo transfigurado de J. V. Foix", Destino, 1413 (1964), 34.

M. Carbonell, “Contribució a l'estudi de les idees polítiques de Foix", Faig (June 1984), 51-59.

M. Carbonell, "Introducció" to J. V. Foix, Obres Completes, III, Articles $i$ assaigs polítics, Barcelona, Edicions 62, 1985. 
M. Carbonell, "Textos de J. V. Foix sobre Ramon Llull", Randa, 17 (1985), 179-193.

J. M. Castellet, "Analogía entre dos poetas", Correo Literario, 5 (1954), 41.

A. Cirici, "J. V. Foix i les arts plàstiques", Serra d'Or, 280 (1983), 19-24.

J. Colomines, preface to J. V. Foix, Catalans ds 1918, Barcelona, Edicions 62, 1965, 5-14.

A. Comas, "On be deixat les claus...: la creació poètica de J. V. Foix", Cap d'Any 1956, Palma de Mallorca, Moll, 1956, 105-112.

P. Crusat, "La poesía de J. V. Foix (En torno a On be deixat les claus...)", Ínsula, 110 (1955), 3-4.

G. Díaz-Plaja, "Ritme i rima a la poesia de J. V. Foix", De literatura catalana. Estudis $i$ interpretacions, Barcelona, Selecta, 1956, 195-207.

G. Díaz-Plaja, "Josep Vicenç Foix", Viatge a L'Atlàntida $i$ retorn a Itaca. Una interpretació de la cultura catalana, Barcelona, Destino, 1962, 135-144.

J. Ferran, J. V. Foix, Madrid, Júcar, 1986.

G. Ferrater, preface to J. V. Foix, Els lloms transparents, Barcelona, Edicions 62, 1969, 7-20.

G. Ferrater, "Nou sonets de Foix comentats", Quaderns Crema, 1 (1979), 43-52.

T. Garcés, "Un historiador i un poeta", Notes sobre poesia, Barcelona, Catalònia, 1933, 90-94.

P. Gimferrer, "Els lloms transparents: J. V. Foix y su doble", Destino, 1965 (1970), 38.

P. Gimferrer, "Foix, nuevamente", Destino, 1702 (1970), 45.

P. Gimferrer, "Descripció d'un Ilibre: Sol, i de dol", Serra d'Or, 160 (1973), 45-47. 
P. Gimferrer, "Notes sobre la poesia en vers de J. V. Foix", , preface to J. V. Foix, Obres Completes, I, Poesia, Barcelona, Edicions 62, 1974, 7-26.

P. Gimferrer, La poesia de J. V. Foix, Barcelona, Edicions 62, 1974.

D. Guansé, "J. V. Foix", Retrats literaris, Mèxic, Catalònia, 1947, 67-71.

J. Junyent, "J. V. Foix, poeta surrealista", Inquietud, 1 (1955), 3-4.

A. Manent, "Els Nadals del poeta J. V. Foix", Condal, Christmas 1954, 45-48.

A. Manent, "Entre la llegenda i la vida de cada dia", Serra d'Or, 160 (January 1973), 20-26.

J. Marco, "M'exalta el nou i m'enamora el vell (Releyendo a J. V. Foix)", Destino, 1.541 (1967), 50.

C. Miralles, "Glossa de Josep V. Foix", in Homenatge a Josep V. Foix amb motiu d'ésser-li conferit el grau de Doctor Honoris Causa, Barcelona, Universitat de Barcelona, 1984.

C. Miralles, "Per ajudar a una lectura de Les irreals omegues", Estudis de literatura catalana en bonor de Josep Romeu Figueras, vol. II, Montserrat, Abadia de Montserrat, 1986, 127-140.

J. Molas, "Del 'Diari 1918' de J. V. Foix", Índice de Artes y Letras, 92 (1956), 21.

J. Molas, "La literatura catalana i els moviments d'avantguarda", L'avenç, 19 (September 1979), 18-26.

J. Molas, "J. V. Foix i l'avantguarda", preface to J. V. Foix, Obres Completes, II, Prosa, Barcelona, Edicions 62, 1979, 7-16.

Ll. Montanyà, "Algunes consideracions sobre la lírica de J. V. Foix", Quaderns de poesia, 7 (1936), 53-57.

D. Oller, "J. V. Foix: els emblemes de la Passió", Serra d'Or, 297 (June 1984), 42. 
M. Ortín, "Sobre Les irreals omegues de J. V. Foix: la densitat expressiva", Quart Creixent, 2.

E. Padrós de Palacios, "J. V. Foix, sempre i ara", Serra d'Or, 2-3 (February-March 1964), 71-72.

E. Padrós de Palacios, "J. V. Foix, intimitat i diàleg", Serra d'Or, 280 (January 1983), 25-27.

J. Palau i Fabre, "J. V. Foix, seny i follia", Quaderns de l'alquimista, Barcelona, Pòrtic, 1976, 147-151.

O. Pi de Cabanyes, "J. V. Foix i la Catalunya ideal", Serra d'Or, 280 (January 1983), 31-33.

J. Pla-Narbona, Escenificació de cinc poemes de J. V. Foix, Barcelona, Editorial Rocas, 1966.

B. Porcel, "J. V. Foix y las realidades fantásticas", Destino, 1540 (1967), 38-39.

A. Puig, "J. V. Foix y su perfil humano", Revista, 500 (1963), 23.

J. Pujol, "Sobre una prosa de Darrer comunicat", Faig (June 1984), 41-48.

J. Romeu i Figueras, "Aproximacions a la poesia de J. V. Foix", preface to J. V. Foix, Del "Diari 1918", Barcelona, Horta ed., 1956, 7-15.

J. Romeu i Figueras, "Nota breu a L'Estrella d'En Perris", Poemes, 7, 1963.

J. Romeu i Figueras, "J. V. Foix, assagista", Serra d'Or, 125 (February 1970), 59-60.

J. Romeu i Figueras, "Darrer comunicat", Serra d'Or, 144 (September 1971), 47-48.

J. Romeu i Figueras, "Comentaris a uns poemes de Les irreals omegues", Serra d'Or, 160 (January 1973), 49-50.

J. Romeu i Figueras, "De mitologia fociana", Presència, 515 (June 1978), 39. 
J. Romeu i Figueras, "Una lectura del primer Nadal de J. V. Foix", Reduccions, 7 (1979), 28-38.

J. Romeu i Figueras, "El component popular en la poesia de J. V. Foix", Serra d'Or, 280 (January 1983), 28-30.

J. Romeu i Figueras, Sobre Maragall, Foix $i$ altres poetes, Barcelona, Ed. Laertes, 1984. It contains all the mentioned articles by J. Romeu i Figueras.

J. Romeu i Figueras, "Sol, i de dol" de J. V. Foix, Barcelona, Editorial Empúries, 1985.

G. E. Sansone, "J, V. Foix, trovatore e surrealista", Studi di filologia catalana, Bari, Adriatica Editrice, 1963, 268-285.

G. E. Sansone, "Nota als preàmbuls poètics de J. V. Foix", Poemes, 7 (1964), 5-6.

R. Santos Torroella, "Mundo poético de J. V. Foix", Correo Literario, 63 (1953), 5.

M. Serrahima, "J. V. Foix en prosa", Serra d'Or, 160 (January 1973), 27.

Several authors, "J. V. Foix: 85 años", Destino, 2.106 (February 1978), 22-32.

E. Sullà, "Tocant a mà...", Serra d'Or, 160 (January 1973), 51.

J. Teixidor, "La poesía de J. V. Foix", Entre les lletres $i$ les arts, Barcelona, J. Horta, ed., 1957, 100-107.

J. Teixidor, "Quatre notes sobre la poesia de J. V. Foix", Els Marges, 7 (1976), 7-12

A. Terry, "Sobre les obres poètiques de J. V. Foix", Serra d'Or, 102 (March 1968), 47-52.

A. Terry, "La idea de l'ordre en la poesia de J. V. Foix", Serra d'Or, 160 (January 1973), 41-43.

J. Triadú, "Presentació oral de J. V. Foix", La literatura catalana i el poble, Barcelona, Selecta, 1961, 103-107.

J. Triadú, "J. V. Foix en la situació de la poesia catalana", Serra d'Or, 160 (January 1973), 29-31. 
Jaume VallcorbaPlana, "Discurs de presentació a la lectura de poemes de J. V. Foix ...", Quaderns Crema, 4 (February 1981), 95-98.

A. Vilanova, "El Diari 1918 de J. V. Foix", Destino, 1.002 (1956), 37. 